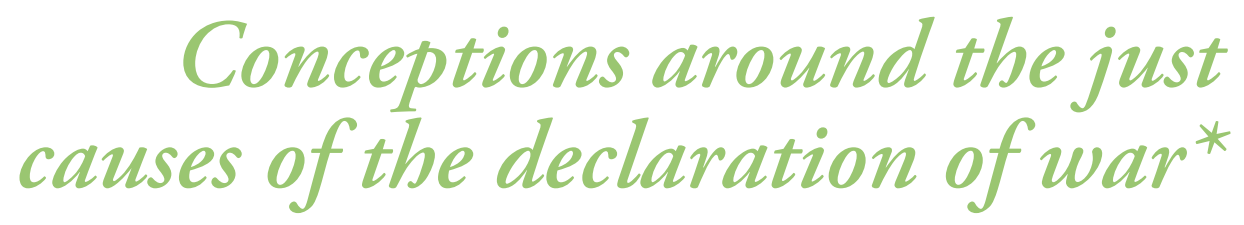

Pp. $10-28$

Juan Carlos Marulanda Hernández ${ }^{\star \star}$

Jaime Yáñez Canal ${ }^{\star \star \star}$

Este artículo deriva de la investigación que integra el cuerpo de trabajos adelantados por el grupo estudios en desarrollo socio-moral, del programa de psicología de la Universidad Nacional de Colombia, dirigido por el profesor Jaime Yáńez-Canal.

** Psicólogo. Magister en psicología de la Universidad Nacional. Docente de la Universidad Nacional de Colombia. Correspondencia: juancmarulandah@gmail.com | ORCID: 0000-0002-3458-1531

*** Psicólogo. Doctor en filosofía de la Katholische Universität Eichstätt. Docente de la Universidad Nacional de Colombia. Correspondencia: jyanezc@unal.edu.co | ORCID: 0000-0001-9839-1123 


\section{Concepción en torno a las causas justas de la declaración de guerra*}

Cómo citar este artículo: Marulanda Hernández, J. C., \& Yáñez Canal, J. (2018). Concepción en torno a las causas justas de la declaración de guerra. Tesis Psicológica, 13(1), 10-28. https://doi. org/10.37511/tesis.v13n1a2

\author{
Recibido: 08 agosto 2017 \\ Revisado: 02 septiembre 2017 \\ Aprobado: 08 junio 2018
}

\begin{abstract}
In order to understand the armed conflict phenomena, some investigations about attitudes and moral judgment towards war have been done from the social psychology field. The present research explores the attitudes and discourses concerning what may be a right circumstance or reason to use military force. A structured questionnaire was built and applied, based on discussions and principles of the just war theory, which is a philosophical approach that considers criteria to legitimize war declarations and regulate military behavior. 169 people were recruited, including demobilized people from illegal armed groups (FARC and AUC), members of armed forces and university students. By means of the data analysis, it is concluded that most of participants agree with justifications of war based on the defense of human rights. At the same time, significant gender, group and educational level differences were found, which allows to claim that there is a relation between the personal and social factors and the concepts and positions towards justice in war.
\end{abstract}

Keywords: war, moral judgment, conceptions of justice.

\section{RESUMEN}

Con el fin de contribuir a la comprensión del fenómeno del conflicto armado desde el campo de la psicología social se han adelantado estudios en los que se indaga por las actitudes y juicios morales frente a la guerra. En el presente artículo se analizan las actitudes y discursos con respecto a los distintos tipos de justificación a los que se ha apelado tradicionalmente para legitimar el uso de la fuerza armada. Para este estudio se construyó y aplicó un cuestionario estructurado basado en las discusiones y planteamientos de la teoría de la guerra justa, una propuesta filosófica que fija criterios para legitimar la declaración de la guerra y establecer límites morales sobre la conducta militar. Se contó con la colaboración de un grupo de 169 personas, integrado por desmovilizados de grupos armados ilegales de Colombia (FARC y AUC), miembros de la fuerza pública y estudiantes universitarios. Tras el análisis de las respuestas, se logra concluir que la mayoría de las personas mantienen una actitud favorable frente a aquellas justificaciones de la guerra que pretenden la defensa de la dignidad humana. Al mismo tiempo, se hallaron diferencias significativas a nivel de género, nivel educativo y grupo poblacional, las cuales permiten establecer la relación entre factores personales y sociales y la divergencia de concepciones y posturas acerca de la justicia en la guerra.

Palabras clave: guerra, juicio moral, concepciones frente a la justicia. 


\section{Introducción}

El debate en torno a los límites en el uso de la guerra ha sido recurrente en la historia del pensamiento político occidental. Tal discusión ha permitido fijar una serie de principios morales y políticos mediante los cuales es posible juzgar la legitimidad del uso de las armas y establecer restricciones al actuar de los combatientes, estos principios conforman la denominada teoría de la guerra justa. Uno de los aspectos tratados en esta teoría es el criterio de causa justa o legítima a partir del cual se valoran las razones o circunstancias que justifican o condenan la declaración de una guerra. Las concepciones sobre lo que constituye una causa justa se han trasformado a lo largo de la historia, la perspectiva inspirada en el liberalismo y la tradición de los derechos humanos son las que cuentan con mayor apoyo en la actualidad. Esta perspectiva juzga como justas solo aquellas guerras que se llevan a cabo en defensa de la dignidad humana o de la libertad y soberanía de las naciones (Walzer, 2001a; Papacchini, 2002; Orend, 2006; Bellamy, 2009).

Si bien los escenarios de guerra y destrucción de los últimos tiempos deberían ser argumentos suficientes para exigir que las naciones apelen a la guerra solo cuando esta sea moralmente justificada, la implementación de este criterio de razón o causa justa en el contexto de la política internacional encuentra todavía algunos obstáculos (Walzer, 2001a, 2001b). De una parte, algunos líderes políticos y militares aún rechazan cualquier intento de limitar las acciones armadas a partir de argumentos morales o legales, al tiempo que declaran que los intereses económicos o ideológicos de un grupo a nación particular son razones legítimas para hacer la guerra. De otra parte, en aquellos escenarios en los que el discurso de la defensa de los derechos humanos es el único que encuentra respaldo, se suele acudir a las diversas interpretaciones o concepciones que pueden tener las personas sobre aquello que es justo o moralmente correcto para enmascarar intereses particulares y manipular la opinión de los ciudadanos (Walzer, 2001a; Papacchini, 2002; Marulanda \& Yáñez-Canal, 2015).

Ya sea gracias al discurso sobre las razones económicas o ideológicas como argumento suficiente para hacer la guerra o mediante el uso o manipulación del lenguaje moral, los líderes políticos o militares han buscado siempre el apoyo de los ciudadanos, pues resulta ser uno de los elementos más importantes para el inició y la continuidad de la guerra. De esta manera, la comprensión de los fenómenos de conflicto y acción armada requiere también una aproximación a la manera en que las personas conciben lo que puede o no ser un argumento moralmente legitimo para declarar la guerra y cómo diversos factores influyen en tales concepciones. La psicología se ha aproximado a este fenómeno desde diversas perspectivas investigativas, las cuales presentan diferencias en cuanto a los enfoques conceptuales y las propuestas metodológicas que plantean.

Una primera propuesta corresponde a los estudios interesados en la medición de actitudes frente a la guerra mediante el uso de instrumentos y escalas psicométricas (Mayton, 1988; Carnagey \& Anderson, 2001; Cohrs \& Mochner, 2002; Cohrs, Moschner, Maes \& Kielman, 2005; Jones, Restori, Howard, Himelfarb \& Boluyt, 2007; Bar-Tal, Sharvit, Halperin \& Zafran, 2012; Bizumic, Stubager, Mellon, van der Linden, Iyer \& Jones, 2013). Este grupo de trabajos se ha enfocado principalmente en el análisis estadístico y la caracterización de las actitudes positivas o negativas de los participantes con respecto a la guerra, que deja en un segundo plano las discusiones sobre las concepciones morales o políticas que puedan dar lugar a dichas actitudes.

Dentro de los temas estudiados destaca la indagación por las diferencias de las actitudes de 
niños y adolescentes de diferentes países frente a la guerra (Schroeder, Gaier \& Holdnack, 1993; Kuterovac, 2000; Myers-Bowman, Walker \& Myers-Walls, 2005; de Souza, Sperb, McCarthy \& Biaggio, 2006; Sarrica \& Wachelke, 2009; Nasie \& Bar-Tal, 2012). También se ha intentado entender la influencia de las emociones sobre actitudes de agrado y desagrado en relación con los conflictos armados (Cheung-Blunden \& Blunden, 2008; Musgrove \& McGarty, 2008). Finalmente, algunas investigaciones han demostrado la relación entre las actitudes frente a la guerra y variables como la orientación política o religiosa (Wille, 2007; Friese, Fishman, Beatson, Sauerwein \& Rip, 2009; Shaw, Quezada \& Zárate, 2011), las diferencias culturales e identificación grupal (van der Linden, Bizumic, Stubager \& Mellon, 2011; Bobowik, Páez, Liu, Licata, Klein \& Basabe, 2014; Li, Li, Huang \& Chiu, 2015) y los rasgos de personalidad (Bothwell \& Kennison, 2004; Lialli \& forsteinsson, 2007; Crowson, 2009; Dunwoody, Plane, Trescher \& Rice, 2014; Sundberg, 2014).

Las investigaciones fundadas en las teorías del desarrollo moral se presentan como una segunda línea de aproximación a la temática planteada. Si bien estos trabajos no abordan directamente la temática de la guerra, contribuyen a su comprensión en tanto abordan el problema del juicio moral en contextos adversos y de violencia. Los trabajos desde este enfoque se fundan en los planteamientos tempranos sobre el desarrollo moral de Piaget (1999) y Kohlberg (1992). Estos autores sugieren un paralelismo entre el desarrollo cognitivo y el del juicio moral, que en sus etapas finales se caracteriza por la capacidad autónoma de emitir juicios fundados en principios universales e independientes de circunstancias o consecuencias particulares (Hoffman, 2002; Yáñez-Canal, 2000). Los abordajes actuales se apoyan en propuestas contemporáneas como la ética del cuidado y la benevolencia de Gilligan y la teoría de los dominios de Turiel, perspectivas que si bien abandonan los esquemas de progreso o evolución psicológica, se apegan a la concepción rawlsiana de justicia como equidad que influenció significativamente la propuesta kolhbergiana (Yáńez-Canal, Perdomo \& Mojica, 2010; Yáñez-Canal \& Mojica, 2012). Otra característica que tienen en común las propuestas tradicionales y contemporáneas en esta línea se relaciona con el uso de dilemas morales y entrevistas semiestructuradas en la indagación sobre el desarrollo de los juicios morales (YáñezCanal, Perdomo \& Mojica, 2010).

Entre los estudios sobre el juicio moral en contextos de violencia resaltan varios trabajos con población infantil y juvenil víctima de la violencia en Colombia, (Riaño, 2002; Echavarría \& Vasco, 2006; Posada \& Wainryb, 2007; Wainryb \& Pasupathi, 2008; Chaux, Arboleda \& Rincón, 2012; Posada, 2012). Los hallazgos logrados a través de estas investigaciones permiten establecer el impacto en la vida de contextos adversos sobre la formulación de justificaciones de actos de agresión y venganza. Al mismo tiempo, concluyen que tales justificaciones no pueden verse como un signo de incomprensión moral o de la noción de justicia, sino que se fundan sobre concepciones alternas en torno a la justicia retributiva o restaurativa y contemplan valores como la fraternidad y la lealtad al grupo. Partiendo de estas investigaciones es también posible reformular las concepciones tradicionales que presentan la incompatibilidad entre el desarrollo moral y las formas de justificación del uso de la violencia. No obstante, queda abierta la pregunta acerca de si tales hallazgos apoyados en ejemplos de violencia entre ciudadanos comunes pueden ser trasladados al espacio de las formas de violencia intergrupal organizada, que caracterizan a la guerra.

Otra perspectiva deabordaje sobrelajustificación de la guerra está integrada por los trabajos sobre

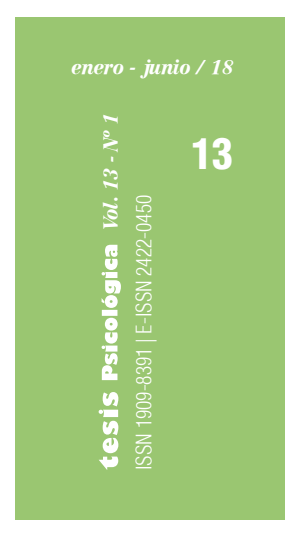


análisis discursivo, en los que se han estudiado los juicios y justificaciones de la guerra consideradas por líderes políticos, mandos militares y combatientes (Moerk, 2002; Hogenraad, 2005; Halversscheid \& Witte, 2008; Gibson, 2012; Pilecki, Muro, Hammack \& Clemons, 2014). Este abordaje indaga por las concepciones sobre valores políticos y morales, discursos emocionales y otros aspectos de la retórica discursiva desde un enfoque interdisciplinar, apoyado en algunos planteamientos de la sociología y la filosofía. Resaltan algunas investigaciones sobre el conflicto colombiano, entre las que cuentan los análisis de los discursos ideológicos que legitiman la violencia (Barreto \& Borja, 2002), los estudios sobre retórica paramilitar y estatal (Botero, 2005; Borja-Orozco, Barreto, Sabucedo \& López-López, 2008) y los estudios sobre discursos emocionales y justificatorios esgrimidos por los actores armados en Colombia (Bolívar, 2006).

Entre los trabajos sobre análisis discursivo llama la atención el estudio de Halverscheid y Witte (2008), en tanto es uno de los pocos trabajos que explora las justificaciones de la guerra y el terrorismo desde la perspectiva de los planteamientos sobre la causa justa y la moralidad de la guerra de la teoría de la guerra justa. En esta investigación se analizaron los discursos públicos de grupos involucrados en algunos conflictos bélicos del siglo XX. Estos discursos fueron categorizados a partir de cuatro posturas éticas: deontológica, utilitarista, intuicionista y hedonista. También se abordaron con más detenimiento las concepciones en torno a la defensa de los derechos de una nación, la reivindicación de los derechos humanos y el respeto a las formas de vida de una comunidad que determinan diversos tipos de justificación de la declaración de guerra. Estas concepciones no pueden ser ordenadas necesariamente según un esquema de estadios progresivos, como usualmente se ha procedido en la psicología del desarrollo moral ni limitarse solo a la exploración de actitudes a partir de pruebas psicométricas. Más bien requieren de una perspectiva integrativa, que contemple las limitaciones y ventajas que ofrece cada vía de investigación ante la complejidad que presenta el fenómeno de la guerra.

En vista de los límites y alcances que presentan las diversas aproximaciones, la presente investigación se plantea con el objetivo de abordar de manera más amplia las concepciones frente a las justificaciones que se suelen esgrimir para declarar la guerra y algunos factores que pueden dar lugar a dichas concepciones. La investigación compara las respuestas y discursos de personas que hicieron parte de grupos armados involucrados en el conflicto colombiano con las posiciones y discursos de aquellos que no han estado involucrados de forma directa en este conflicto. Para ello, se toma como marco conceptual para el análisis el conjunto de reflexiones filosóficas sobre la justicia en la guerra, como una concepción de lo moral que amplía los horizontes de los planteamientos tradicionales de la psicología del desarrollo moral. Dado que es una primera aproximación al problema de las concepciones de la justicia de la guerra con población colombiana y que se parte de un marco conceptual distinto al que han considerado otras líneas de investigación, el presente trabajo se limita a caracterizar las concepciones y discursos aportados por los participantes. Las investigaciones mencionadas anteriormente han sido consideradas en la propuesta de un diseño metodológico que combina la estrategia de medición de actitudes frente a distintos tipos de justificación y el análisis de los discursos o afirmaciones que pueden acompañar las actitudes expresadas. Con todo ello se pretende aportar nuevos elementos para la discusión en el campo de investigación y generar nuevas rutas de investigación en las que sea posible la integración de diversos elementos metodológicos y conceptuales para la comprensión de la guerra y los conflictos armados. 


\section{Método}

\section{Diseño de investigación}

El estudio propuesto es de carácter no experimental. Su pretensión es explorar y describir las concepciones que tiene las personas frente a lo que puede o no ser una razón justa para hacer uso de la guerra, mediante empleo de un cuestionario estructurado y el análisis de respuestas complementarias de cada ítem.

\section{Participantes}

El estudio contó con la colaboración de 169 personas de la ciudad de Bogotá (119 hombres y 50 mujeres $M=25.4$ años, rango de edad 1658 años), pertenecientes a cinco grupos poblacionales: 33 desmovilizados de grupos insurgentes, 31 desmovilizados de grupos paramilitares o de autodefensa, 14 oficiales de la policía metropolitana de Bogotá, 37 patrulleros en formación de la Escuela de Policía Metropolitana de Bogotá y 54 estudiantes universitarios de primer año de psicología.

La selección de los grupos se hizo con el objetivo de explorar las actitudes de personas que hubiesen tenido una relación directa con el conflicto armado o contexto de violencia armada en Colombia. Se escogió también un grupo de estudiantes con el fin de realizar comparaciones con respecto a las posiciones de aquellos participantes que no hubiesen tenido contacto con el conflicto armado. El tamaño y selección del grupo de participantes dependió de la posibilidad de acceder al tipo de población esperada, de manera que el número y características de los participantes estuvo determinado por las disposiciones de las instituciones y la disponibilidad de las personas en los tiempos de aplicación. En el caso de los excombatientes de grupos alzados en armas y los miembros de la fuerza pública, el número de participantes fue fijado por los criterios de acceso establecidos por las instituciones, considerando que por cuestiones de logística y seguridad dichas instituciones tienden a mantener criterios estrictos para el desarrollo de investigaciones. El número de los participantes universitarios se determinó teniendo en cuenta el tamaño de los grupos restantes. A pesar de las dificultades, se considera que el tamaño de la muestra permite un análisis riguroso y la presentación de conclusiones relevantes sobre los supuestos teóricos planteados.

\section{Instrumento}

Las actitudes y concepciones de los participantes frente a las justificaciones de la guerra fueron recolectadas a través de un cuestionario en el que los participantes debían mostrar su actitud, a través de una escala de acuerdodesacuerdo ante diferentes tipos de razones o justificaciones para la declaración de la guerra. Las justificaciones estuvieron basadas en diversos tipos de argumentos y circunstancias propias de los conflictos armados contemporáneos que han sido tenidos en cuenta dentro de la tradición de la guerra justa: las justificaciones basadas en el interés económico, la apelación al legalismo internacional, los objetivos terroristas, la disuasión nuclear, la guerra irregular y la intervención humanitaria, entre otros (Walzer, 2001a; Orend, 2006; Bellamy, 2009).

La construcción y validación del instrumento se realizó gracias al juicio de expertos en las aéreas de psicometría, filosofía política y estudios sobre conflicto armado de la Universidad Nacional de Colombia. Se tomaron en cuenta las observaciones sobre redacción, pertinencia y fundamentación conceptual de cada uno de los ítems para la modificación del instrumento preliminar con el fin de que fuese coherente con los objetivos de la investigación. Los enunciados 
también fueron revisados y corregidos partiendo de los resultados de una prueba piloto realizada con 13 participantes (7 hombres y 6 mujeres), seleccionados a partir de las características socioeconómicas y de nivel educativo del grupo de participantes al que se aplicaría el instrumento final.

Se realizó un análisis de fiabilidad del instrumento con los ítems agrupados en las cinco categorías que se presentan en la sección de análisis de datos. El instrumento presentó una consistencia interna con un alfa de Crombach $=0.57$. Aunque dicho valor puede ser considerado como bajo dada la validación teórica apoyada en expertos, el carácter novedoso del estudio y la extensión del cuestionario, se considera como un instrumento valido para soportar las conclusiones planteadas a partir del análisis por categorías. En los demás casos, y cómo se puede observar en el apartado de resultados, el análisis se hizo de manera particular considerando por separado cada uno de los tipos de justificación propuestos en cada ítem.

\section{Procedimiento}

La aplicación del cuestionario se realizó de manera presencial, utilizando la versión impresa del instrumento. En un primer momento se ofreció la información sobre los objetivos y alcances de la investigación y se solicitó el consentimiento de los participantes. Posteriormente se requirió la información personal del participante y luego se dieron las instrucciones sobre el diligenciamiento del cuestionario. Se les explicó que no existía una respuesta correcta y que debían contestar el cuestionario basados en sus propias concepciones. También se indicó que podían complementar sus respuestas usando los espacios en blanco anexos a cada ítem. El rol del investigador se limitó a la aclaración de dudas relacionadas con el diligenciamiento del cuestionario, con el fin de evitar cualquier sesgo en las respuestas expresadas.
Dadas las características particulares de los participantes del grupo de desmovilizados y las dificultades en cuanto a espacio, logística y seguridad que presentan los centros de atención a excombatientes, algunas aplicaciones del cuestionario se realizaron de manera individual y en la mayoría de los casos en grupos reducidos de no más de cinco personas. En cuanto a los demás grupos, fue posible aplicar el instrumento en grupos más amplios, no mayores a veinte personas. La aplicación de los cuestionarios se hizo respetando las condiciones impuestas por las instituciones y las recomendaciones para la investigación en seres humanos establecidas por los códigos que reglamentan el ejercicio de la profesión del psicólogo y su actividad investigativa en Colombia.

\section{Análisis de los datos}

Luego de obtener las frecuencias generales de respuestas para cada enunciado, se exploró la relación entre las diferentes variables (población, género y nivel educativo) y las frecuencias de respuesta para cada uno de los enunciados a partir de la prueba de independencia $\chi 2$. Posteriormente se analizaron las respuestas agrupadas en cinco categorías: (a) búsqueda de beneficio o bienestar económico; (b) defensa ante un posible dańo o perjuicio contra un Estado; (c) defensa de los derechos dentro de un sistema político particular; (d) reivindicación de principios morales universales o defensa de derechos humanos, y (e) guerra como vía para la resolución del conflicto interno. Se evaluaron las diferencias entre grupos mediante dos pruebas estadísticas no paramétricas: Kruskal Wallis y Prueba U de MannWhitney.

Para el análisis de las respuestas complementarias a las escalas actitudinales se tuvo en cuenta la técnica de análisis de contenido descrito en Bardín (1996) y Giroux y Tremblay (2004) y los ejemplos de análisis discursivo de los trabajos de 
Bolívar (2006) y Halversscheid y Witte (2008). Las respuestas complementarias tenían un carácter opcional y la mayoría de los participantes prefirió no complementar o justificar la actitud evaluada frente a cada una de las justificaciones de la guerra incluidas en el cuestionario. Por tal razón, del total de respuestas complementarias esperadas se obtuvo solo entre un $19.5 \%$ y un $44.9 \%$ de las respuestas, dependiendo de cada uno de los enunciados.

Las respuestas se clasificaron con base en dos dimensiones derivadas de la discusión conceptual: (I) juicios morales o políticos frente a la guerra y (II) representación de los actores o grupos involucrados en un conflicto armado: Estado, fuerzas armadas y grupos armados ilegales, entre otros. Teniendo en cuenta estas dos dimensiones iniciales, se plantearon seis categorías distintas de análisis: (a) juicios u opiniones con respecto al proceder de las fuerzas armadas y los grupos armados involucrados en el conflicto; (b) juicios u opiniones con respecto al Estado (en su sentido más general, más allá de su participación a través de sus fuerzas militares), sus deberes y funciones; (c) juicios $\mathrm{u}$ opiniones con respecto al papel de los organismos internacionales; (d) juicios morales y/o políticos que legitiman o condenan el uso de la fuerza; (e) enunciados en los que se plantean consideraciones sobre momentos y circunstancias apropiadas para emplear la fuerza (categoría emergente tras el análisis); (f) enunciados en los que se exponen alternativas distintas al uso de la violencia (categoría emergente).

\section{Resultados}

El presente apartado está dividido en dos subsecciones. En la primera se presentan los resultados logrados a partir del análisis estadístico de las respuestas a las escalas de actitud frente a las afirmaciones que justifican el inicio de una guerra. Se describen los porcentajes de respuesta para cada uno de los enunciados y los valores correlacionales que muestran la asociación entre las características de la población y el tipo de actitudes expresadas.

En la segunda parte se resumen los hallazgos encontrados gracias al análisis discursivo de las respuestas que complementaban las actitudes expresadas. Se presentan algunos ejemplos y observaciones sobre las afirmaciones expresadas por los participantes ante cada una de las justificaciones de la guerra, clasificadas a partir de las categorías antes descritas.

\section{Actitudes frente a las condiciones que justifican el inicio de una guerra}

Los porcentajes de frecuencia de las respuestas para cada una de las razones que justifican la guerra ofrecen una primera idea acerca del tipo de situaciones que los participantes consideran como causas legítimas para la declaración de una guerra. Los porcentajes presentados en la tabla 1 muestran que la mayoría de los encuestados expresaron una actitud de desacuerdo con respecto a la guerra como una vía de solución ante los diferentes tipos de conflictos entre naciones o grupos sociales. De igual manera, las actitudes de rechazo frente al uso de la guerra fueron mayores ante las justificaciones basadas en la búsqueda de un beneficio de tipo económico, mientras que hubo mayores porcentajes de aprobación ante la justificación del uso de las armas cuando su objetivo es la defensa de los derechos humanos y la lucha por implantar un sistema democrático. 
TABLA 1. Actitudes frente a las justificaciones de la guerra (porcentajes)

\begin{tabular}{|c|c|c|c|c|}
\hline Criterio de justificación & $\begin{array}{c}\text { Total } \\
\text { desacuerdo }\end{array}$ & $\begin{array}{l}\text { Mediano } \\
\text { Desacuerdo }\end{array}$ & $\begin{array}{l}\text { Mediano } \\
\text { acuerdo }\end{array}$ & Total acuerdo \\
\hline $\begin{array}{l}\text { 1. Lograr el control de recursos naturales y rutas } \\
\text { económicas }\end{array}$ & 59.9 & 24.0 & 10.2 & 6.0 \\
\hline 2. Castigar el rompimiento de relaciones económicas & 71.6 & 19.5 & 1.8 & 7.1 \\
\hline 3. Evitar el monopolio de avances tecnológicos y científicos & 38.0 & 28.3 & 22.3 & 11.4 \\
\hline 4. Refrenar políticas agresivas y armamentistas & 35.7 & 29.2 & 25.0 & 10.1 \\
\hline 5. Detener el desarrollo de armamento nuclear & 40.1 & 18.0 & 20.4 & 21.6 \\
\hline 6. Defender el territorio de invasiones & 38.7 & 23.8 & 23.2 & 14.3 \\
\hline $\begin{array}{l}\text { 7. Derrocar un tirano que comete crímenes de lesa } \\
\text { humanidad }\end{array}$ & 21.6 & 21.6 & 26.9 & 29.9 \\
\hline $\begin{array}{l}\text { 8. Evitar la persecución y el exterminio de una raza, religión } \\
0 \text { cultura }\end{array}$ & 49.1 & 10.2 & 20.4 & 20.4 \\
\hline 9. Implantar un sistema democrático & 30.5 & 19.8 & 25.7 & 24.0 \\
\hline 10. Lograr la equidad y la repartición igualitaria de riqueza & 48.2 & 28.6 & 13.7 & 9.5 \\
\hline $\begin{array}{l}\text { 11. Garantizar el establecimiento de la propiedad privada y } \\
\text { libertad económica }\end{array}$ & 31.0 & 33.3 & 20.2 & 15.5 \\
\hline $\begin{array}{l}\text { 12. Detener las agresiones e injusticias cometidas por un } \\
\text { sector de la población }\end{array}$ & 29.3 & 18.0 & 32.3 & 20.4 \\
\hline
\end{tabular}

Nota: los doce criterios de justificación expuestos dentro del cuestionario se derivan de las razones que han sido consideradas como legítimas por parte de las diversas posturas que han integrado la tradición de la guerra justa. A su vez, cada criterio de justificación se relaciona con circunstancias o problemáticas que conducen a la guerra.

\section{Diferencias en actitudes entre hombres y mujeres}

La diferencia entre hombres y mujeres con respecto a las actitudes de acuerdo y desacuerdo es notoria frente al enunciado en el que se justifica la guerra como forma de defensa del territorio: el porcentaje de desacuerdo fue mayor en el grupo de las mujeres $(74 \%)$, en comparación con el de los hombres (58.1\%). Dicha diferencia se sustenta tras el análisis mediante prueba de independencia con $\chi 2(3.167)=8.609 \mathrm{p}<.050]$.

\section{Diferencias en actitudes con relación al nivel educativo de los participantes}

Las diferencias en cuanto al nivel educativo solo fueron determinantes en la respuesta al enunciando que proponía la guerra como forma de castigo contra una nación que había roto relaciones económicas con nuestro país. A mayor nivel educativo, mayores fueron los porcentajes de respuestas en la opción de total desacuerdo. También se observa que los participantes que habían cursado solo primaria presentaron una tendencia mayor al acuerdo (32\%), mientras los demás grupos manifestaron un rechazo por encima del $90 \%$. Tras la prueba de independencia $\chi 2$ se prueba la relación entre la variable grupo y la repuesta a este ítem $[\chi 2(6.163)=14.58 \mathrm{p}<.05]$.

\section{Diferencias en las actitudes en relación con el grupo poblacional}

Tras el análisis mediante prueba de independencia $\chi^{2}$, se logró sustentar la relación significativa de la pertenencia a uno de los grupos de participantes y los siguientes seis tipos de justificación: (a) lograr el control de recursos naturales y rutas económicas $\left[\chi^{2}(12.167)=33.933, \mathrm{p}<.050\right]$; (b) castigar el rompimiento de relaciones económicas, $\left[\chi^{2}(12.169)=29.390 \mathrm{p}<.050\right]$; (c) 
$\left[\chi^{2}(12.166)=22.336 \mathrm{p}<.050\right]$; (c) evitar el monopolio de avances tecnológicos y científicos; (d) evitar la persecución y el exterminio de una raza, religión o cultura $\left[\chi^{2}=(12.167)=25.938 p\right.$ $<.050]$; (e) detener las agresiones e injusticias cometidas por un sector dela población $\left[\chi^{2}(12.167)\right.$ $=27.970 \mathrm{p}<.050]$, y (f) lograr la equidad y la repartición igualitaria de riqueza $\left[\chi^{2}=(12.168)=\right.$ $27.671 \mathrm{p}<.050]$.
En la tabla 2 es posible observar los porcentajes de respuesta de cada grupo frente a los seis ítems en los que se evidenció una relación con la pertenencía a un grupo determinado. Basados en tales porcentajes, resultan notorias las diferencias de los desmovilizados de grupos armados ilegales con respecto a los demás grupos en cuanto a las justificaciones apoyadas en intereses económicos, que muestra una mayor tendencia a aceptar este tipo de razones para hacer la guerra.

TABLA 2. Porcentajes de respuestas favorables por grupo frente a algunas justificaciones de la guerra.

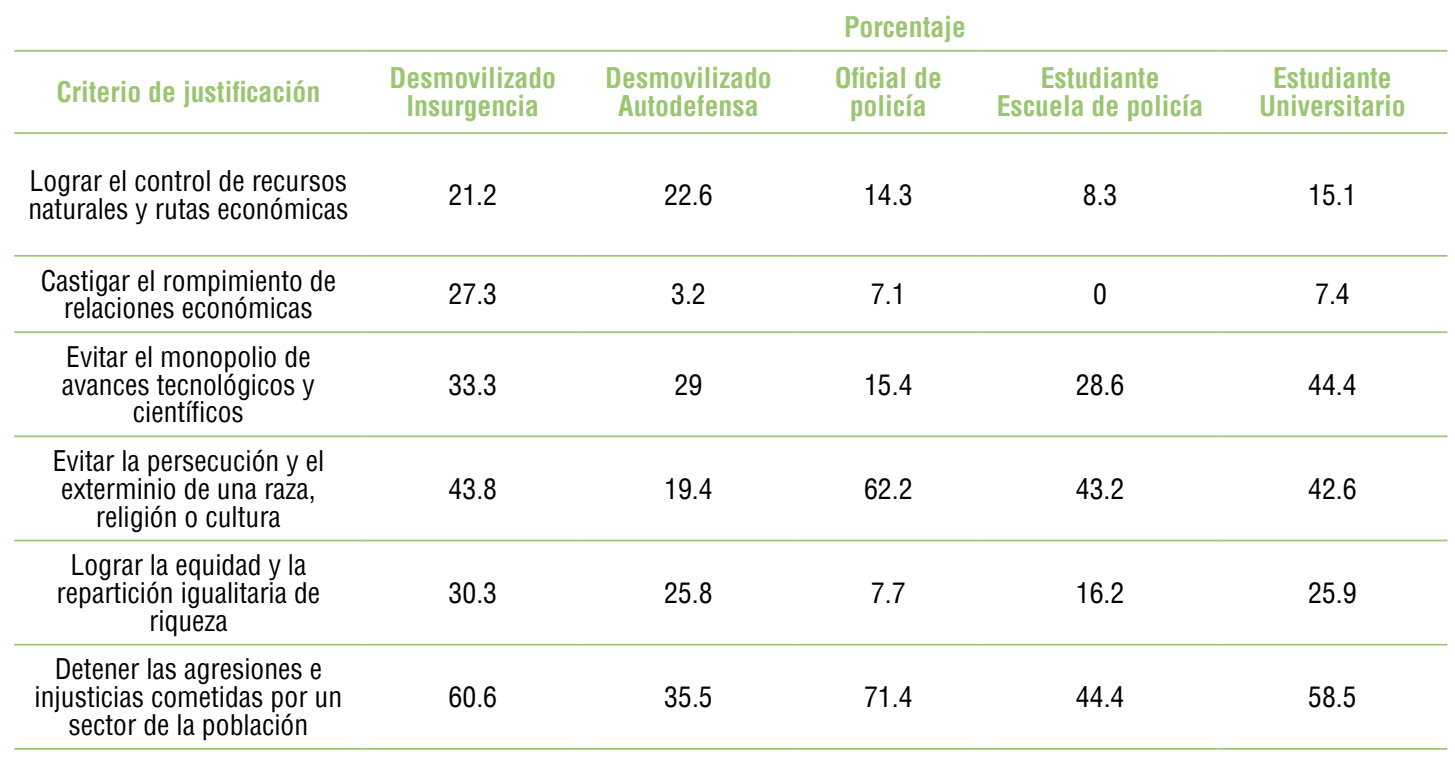

Fuente: Autores

Finalmente, con el fin de observar diferencias en las tendencias de respuestas frente a los ítems agrupados en las cinco grandes categorías, se realizó un análisis mediante la prueba KruskalWallis. La calificación para cada categoría se obtuvo mediante la sumatoria de los puntajes de dichas categorías, establecidos en una escala de 4 (extremo desacuerdo) hasta 16 (extremo acuerdo). En la primera de estas dos categorías, el grupo de desmovilizados de la insurgencia mostró una mayor aceptación de las justificaciones basadas en beneficios económicos con un puntaje promedio de 8.4, en comparación con el grupo de estudiantes de la Escuela Metropolitana de Policía (puntaje promedio=6.3), el grupo de oficiales de la misma institución (puntaje promedio= 6.8) y el grupo de desmovilizados de las autodefensas (puntaje promedio=6.9). En el caso de la categoría referente al tema de las guerras al interior de una nación, se evidenció una diferencia significativa principalmente entre los puntajes de los desmovilizados de la insurgencia con un puntaje promedio de 11 , con lo cual se muestra que hay un mayor grado de aprobación 
ante este tipo de guerras con respecto a los desmovilizados de los grupos de autodefensas, con un puntaje promedio de 8 .

Tras hacer el análisis mediante prueba de independencia $\chi^{2}$ para determinar la relación entre la pertenencia a uno de los grupos y las respuestas a los ítems agrupados por categorías, se observó que hay diferencia entre los grupos en cuanto al conjunto de enunciados que planteaban la búsqueda de un beneficio o bienestar económico $[\chi 2=9.80$ con $4 \mathrm{gl}$; sig. $0.044<0.05]$, y el conjunto de enunciados que plantean la guerra como una vía para la resolución del conflicto generado al interior de una sociedad [ $\chi 2=9718$, con 4 gl. sig. $0.045<0.05]$.

Discursos en torno a la justificación de la guerra

Como se mencionó en el apartado en el que se describe el procedimiento de análisis de los datos, la mayoría de los participantes prefirió no dar respuestas complementarias. Por ello no fue posible hacer un análisis exhaustivo que permitiese la exposición de indicadores cuantitativos o que ilustrase patrones de distribución de frecuencias de respuesta, opiniones y uso de lenguaje particular entre los grupos, estrategias habituales en algunos de los estudios que siguen el enfoque de análisis discursivo. Por tal razón, como complemento a los análisis estadísticos, los hallazgos aquí presentados responden a una caracterización general de los rasgos significativos presentes en los discursos de los distintos grupos participantes apoyado en las respuestas de algunos de sus integrantes. La presente exposición se acompaña de algunos ejemplos representativos de las respuestas, encerrados entre comillas, con el fin de ilustrar los modos propios de expresión de los participantes.

Para empezar, cabe destacar las reacciones de rechazo por parte de los desmovilizados de grupos armados ilegales frente a las justificaciones de la guerra fundadas en el beneficio económico que genera el control de recursos naturales y rutas comerciales. En los discursos de algunos de estos participantes se arguye la defensa de los derechos inherentes a todo Estado tales como la autonomía, la soberanía y la defensa del territorio: "la soberanía es invulnerable sin excepción bajo ninguna excusa" (desmovilizado de autodefensas); "cada país es independiente de los demás, cada país tiene su presidente y las ramas de un Estado que pueden hacer lo que mejor les convenga" (desmovilizado de grupo insurgente). En otros casos se estableció una analogía entre el espacio doméstico o el hogar y el territorio nacional y sus fronteras: "estaría de acuerdo porque cuando a uno se le meten en la casa no le queda más nada que responder" (desmovilizado de grupo insurgente). De otro lado, en los discursos de los participantes de los demás grupos, el rechazo a guerras con propósitos económicos se apoyó en consideraciones sobre el respeto a los derechos humanos o la defensa de valores como la calidad de vida, la tranquilidad, la vida de una persona o comunidad y el respeto a leyes y normas, que primaban sobre la riqueza, los recursos naturales o cualquier otro tipo de bien material.

La defensa del territorio y la soberanía dio lugar a otro tipo de discusión, en la que el tema fueron las circunstancias que daban lugar a conflictos sustentados en la invasión o violación de las fronteras entre naciones. Parte de los estudiantes universitarios criticaron la existencia de fronteras y las restricciones de movilidad internacional, arguyendo que son disposiciones que solo benefician intereses particulares: "todos debemos tener la libertad de viajar a otros países". Por otra parte, voluntarios vinculados a la escuela de policía enlazaron el problema de la invasión del territorio con los actos de patrocinio de organizaciones terroristas por parte de gobiernos extranjeros. 
Algunos participantes desmovilizados de grupos insurgentes, estudiantes de la escuela de policía y estudiantes universitarios expresaron varias razones para defender el uso de la fuerza cuando su objetivo era evitar el monopolio tecnológico y científico. Varios de sus argumentos se caracterizaron por establecer una relación entre el acceso a los recursos tecnológicos y científicos y el respeto a derechos fundamentales de los individuos. En otros casos, el acceso a este tipo de recursos se presenta como garantía de bienestar comunitario y progreso social. Entre los estudiantes universitarios fue más frecuente y explicita la apelación a valores como la vida y la libertad: "pues se buscaría los medios para acceder a los medicamentos que curen enfermedad letal ya que si fueran muchas las victimas prima el bien común sobre el particular y prima el derecho a la vida" (estudiante Escuela Metropolitana de Policía). La apelación a estos valores se puede interpretar también como una forma de solucionar el dilema en torno al bienestar general y el bienestar particular.

El conflicto en torno al monopolio de recursos científicos y tecnológicos generó otro tipo de respuestas, particularmente entre desmovilizados de grupos armados y universitarios, que consideraron como una obligación del Estado garantizar la satisfacción de necesidades primordiales y el disfrute de los bienes básicos, así como el acceso a recursos tecnológicos: "porque un gobierno que no deja que el pueblo utilice lo que es del pueblo no es un gobierno que deba estar en el gobierno" (estudiante universitario). En relación con el apartado anterior, llama la atención la tendencia de estos grupos a aceptar las guerras justificadas en este argumento, lo que sugiere que conciben el acceso a la tecnología como parte del bienestar general y un derecho que debería ser garantizado por el Estado. Algunos participantes manifestaron que cuando un mandatario elude esta obligación debería ser derrocado, ya sea a través de medios democráticos o, según la opinión de un grupo pequeño de participantes, mediante el uso de la fuerza.

A excepción de los desmovilizados de grupos de autodefensas, en las respuestas de los distintos grupos se consideró la carrera armamentista y la estrategia de disuasión nuclear como un riesgo para la humanidad, que debía ser evitado por medio del uso de la fuerza: "que no se debe permitir en ningún lugar del planeta esta práctica que atenta contra la estabilidad del mundo y de la raza humana" (desmovilizados de grupos insurgentes). También se mencionó como un tema problemático el gasto que hacen las naciones en la construcción de este tipo de materiales al tiempo que se desatienden cuestiones prioritarias como la educación y la satisfacción de necesidades básicas.

En relación con los enunciados en que se justificaba la guerra en favor de sistemas democráticos, los desmovilizados de grupos insurgentes, los estudiantes de la escuela de policía y los estudiantes universitarios plantearon la necesidad de defender la democracia, pues es vista como un modelo de gobierno que reivindica distintos valores como la libertad, la igualdad, la libre expresión, el progreso y el bienestar.

De otra parte, fueron muy pocos los comentarios en los que se defendía el uso de la violencia comprometida con la defensa de la equidad social o de las libertades económicas, valores también asociados a los sistemas democráticos. Algunos desmovilizados de grupos armados apoyaron este tipo de guerra y consideraron que hay un uso legítimo de la fuerza cuando el gobierno incumple con su función de protección y preservación del bienestar del ciudadano. En este caso en particular, se reprocha el abuso del poder, la no garantía de la participación popular y el favorecimiento de intereses particulares como faltas que justifican la rebelión:

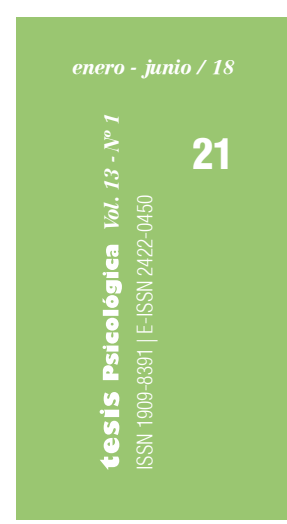


"si el presidente es un tirano al pueblo le toca revelarse" (desmovilizado de autodefensas). Los demás participantes encontraban a este tipo de problemáticas una solución no violenta a través de transformaciones de la política nacional.

Por último, se examinaron las reacciones frente aquellos conflictos que involucran crímenes de lesa humanidad, genocidio, exterminio cultural o ataques a comunidades específicas. Estos últimos ítems guardaban relación con el razonamiento de la emergencia suprema propuesto dentro de la teoría de la guerra justa, que plantea que el ejercicio de la fuerza resulta justo solo en aquellos casos en que la vida de una comunidad se encuentre en peligro o se violente gravemente la dignidad humana. Frente al caso que plantea el derrocamiento de un gobernante que comete crímenes de lesa humanidad, se plantearon dos tipos de argumentos que discutían sobre la posibilidad de una intervención internacional con propósitos humanitarios. Algunos participantes de los distintos grupos se manifestaron en contra de esta idea : "sería brindarle apoyo pero cada quien mirará como solucionan sus problemas es como dicen por ahí la ropa sucia se lava en casa" (estudiante Escuela Metropolitana de Policía); "no meterse en gobierno que no les pertenece" (estudiante universitario). En otras respuestas se planteó la necesidad de este tipo de acciones: "no permitir que una persona sobrepase sus límites de poder" (desmovilizado de grupo insurgente); "porque la guerra no solo sirve para hacer daño también para defender a los que lo necesitan" (estudiante universitario). Mientras las reacciones en contra de la intervención favorecen la idea del respeto de la soberanía y la autodeterminación, los discursos a favor critican el abuso del poder y eventualmente llaman a la cooperación entre naciones o sociedades. Este tipo de intervenciones armadas fueron defendidas por los participantes vinculados a la Escuela Metropolitana de Policía y los estudiantes considerando el respeto a la vida: "la vida del ser humano prima sobre estos dictadores y monarquías" (oficial de policía); "está atentando con la vida y tranquilidad del ser humano" (estudiante universitario). En estos argumentos, el valor de la vida se ubica por encima de cualquier otro valor o ideal, presentándose como un derecho fundamental e inherente a todo ser humano. De allí que bien sea en este caso o en cualquier otro que se encuentre bajo amenaza, los participantes consideren la defensa de la vida algo insoslayable.

En contraste con el caso anterior, no se evidenció una división tajante entre los participantes con respecto a sus reacciones frente a la justificación de las guerras cuyo fin es detener abusos y crímenes contra la dignidad humana. La mayoría expresó comentarios en favor de este tipo de guerra con argumentos en los que se empleó un lenguaje que apelaba a valores morales y reclamaba el respeto a los derechos humanos. Para defender la guerra ante la persecución racial o religiosa, los participantes de los diferentes grupos esgrimieron argumentos en favor de la igualdad, libertad de culto y la libre expresión: "todos tenemos el libre albedrio y somos autónomos de elegir nuestra religión" (desmovilizado de autodefensas); "la diversidad étnica es un derecho, ya nos encontramos en una era donde se le respeta esto, la era de Hitler ya pasó" (estudiante Escuela Metropolitana de Policía). De igual manera, para justificar la guerra en defensa de un sector de la población que ha sido víctima de injusticia por quienes ostentan el poder político o económico, varios participantes de los distintos grupos acudieron al lenguaje de los derechos y enfatizaron el valor de la libertad y la censura a la esclavitud: "nadie tiene derecho a ser esclavo ni a injusticias por parte del gobierno" (estudiante universitario); "nadie tiene la potestad y el derecho de hacer esclavo a alguien ni por más autoridad que este tenga” (oficial Escuela Metropolitana de Policía). 


\section{Discusión}

Después de la exposición de resultados conviene volver sobre algunos apartados que merecen comentarios adicionales. El hallazgo más significativo tiene que ver con la marcada tendencia hacia la aceptación de aquellas justificaciones que enfatizan en la defensa de principios humanitarios, lo que a su vez supone un rechazo generalizado hacia las razones que benefician interés económico o bienes materiales y de forma menos radical hacia aquellas que procuran la protección del Estado y sus derechos en el ámbito legal internacional. Esto supone que para las personas no todas las guerras pueden aceptarse o rechazarse de forma absoluta y que nuestros juicios frente a la guerra parten de nuestras consideraciones sobre principios de moralidad sustentados en la ética de los derechos humanos.

La guerra es siempre analizada en términos de los intereses o valores que buscan defenderse, aun cuando esa defensa implica el causar daño o destrucción. Obviamente, las posturas frente a estos valores o intereses son divergentes y dependientes de los cambios históricos o las diferencias culturales. De cualquier modo, ninguna guerra ha sido justificada apelando a algo diferente de aquello que se piense como favorable para el ser humano y su sociedad. Como se evidencia en investigaciones anteriores sobre el juicio moral frente al uso de la violencia (Echavarría \& Vasco, 2006; Posada \& Wainryb, 2007; Wainryb \& Pasupathi, 2008; Posada, 2012), los resultados del presente estudio también evidencian una prevalencia del lenguaje moral en las reflexiones acerca de la justicia en la guerra, no solo a través de las actitudes frente a los distintos tipos de justificaciones incluidas en el cuestionario, sino también en las argumentaciones construidas por algunos participantes. No obstante, el rechazo general a la mayoría de las justificaciones evidencia cómo algunos discurso políticos e ideológicos han perdido su vigencia y legitimidad, mientras el discurso sobre la defensa de la dignidad humana se acoge cada vez más como la única razón legítima para acudir a las armas. Tal hallazgo sobre el cambio del discurso político es consistente con el análisis de discursos oficiales realizado por Halverscheid y Witte (2008), en el que las justificaciones de tipo moral presentan una mayor frecuencia en comparación con otro tipo de discursos justificatorios.

En los casos que planteaban una justificación fundada en la defensa de principios humanitarios, la apelación al lenguaje moral fue un rasgo característico principalmente de los grupos vinculados a la Escuela Metropolitana de Policía y los estudiantes universitarios. Entre los desmovilizados también hubo este tipo de argumentaciones, aunque fue más marcada la tendencia a expresar enunciados apoyados en otro tipo de consideraciones. La importancia de la vida fue el argumento más recurrente en los discursos, sobre todo para rechazar las guerras que persiguen intereses económicos y para defender el uso de la fuerza contra la proliferación de armas de destrucción masiva, el abuso de poder por parte de dictadores, detener los crímenes contra la humanidad y defender un sistema político como la democracia. Además de acudir al derecho a la vida, los participantes mencionaron valores como la libertad y la igualdad. No obstante, es notorio como a lo largo del cuestionario ambos conceptos tomaban diversos significados entre las repuestas de los participantes de los diferentes grupos. La libertad apareció a veces como opuesta a la esclavitud, otras veces se enunció para hablar de la libre expresión de la opinión y finalmente se entendió en relación con la elección de religión o el desarrollo de la identidad. En el caso de la igualdad, se presentó a veces como valor contrapuesto a la

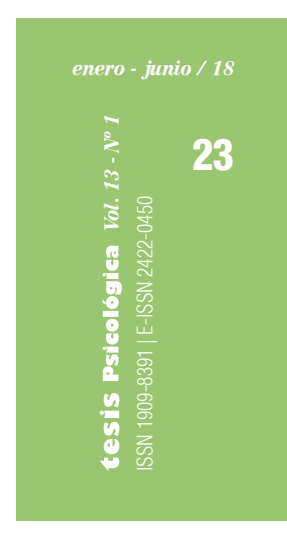


discriminación racial o cultural y en otros guardó relación con la idea de la justicia distributiva y la equidad.

Es posible suponer que este cambio de significado esté sujeto a las diversas concepciones de lo que es justo o correcto para cada persona y al contexto de discusión en que se emplea. Este es un aspecto que no fue posible explorar a fondo dado el tipo de información disponible.

Es importante también considerar la diferencia entre los grupos en cuanto a las posiciones asumidas frente a las obligaciones del Estado. En los discursos analizados, el Estado fue presentado como el garante del acceso a recursos y la calidad de vida de los habitantes de una nación. A su vez, se le adjudicó el deber de velar por el orden y el cumplimiento de la ley mediante el uso justo de su poder. Frente a tales funciones, los participantes desmovilizados de grupos insurgentes, los estudiantes de la Escuela Metropolitana de Policía y los estudiantes universitarios mencionaron el incumplimiento de estas funciones y arguyeron que en muchos casos el Estado favorecía los intereses de una clase privilegiada y abusaba de su poder, lo cual justificaba para algunos la búsqueda de un cambio a través de la violencia. De otro lado, buena parte de los desmovilizados de grupos de autodefensas y oficiales de policía emitieron argumentos en favor del respeto ante las disposiciones legales y el mantenimiento del orden. Estas diferencias entre posturas en favor o en contra del estatus quo pueden ser explicadas no solo a partir de la brecha generacional que diferenciaba a algunos de los grupos, sino también por el rol que ciertas instituciones u organizaciones han cumplido en la vida nacional y que ha determinado sus posiciones políticas. Las diferencias en estos discursos guardan similitud con los trabajos previos con población colombiana, en los que se presentan las diferencias políticas entre líderes y combatientes de distintos grupos (Bolívar, 2006; Borja-Orozco, Barreto, Sabucedo \& López López, 2008). En ambos casos es posible suponer que las concepciones sobre las razones justas para hacer la guerra están vinculadas a la pertenencia y afinidad política o ideológica.

Por último, y con el ánimo de orientar futuros abordajes, es importante mencionar algunas limitaciones de la presente investigación. De una parte, las condiciones del proceso de reintegración y el control institucional, así como la desconfianza por parte de algunos excombatientes frente a la investigación, dificultaron la recolección de información. La construcción del cuestionario tuvo como uno de sus objetivos sortear este tipo de dificultades, aunque esto implicó el no poder profundizar como se esperaba en concepciones particulares sobre la justicia, la guerra y los derechos humanos. De otro lado, las conclusiones del estudio acerca de las actitudes de miembros de grupos armados ilegales deben interpretarse teniendo en cuenta que no fue posible indagar por las concepciones de combatientes activos y en condiciones de vida ilegal, cuyas respuestas podrían diferir de las de los excombatientes sometidos a un proceso de reintegración. 


\section{Referencias}

Bardín, L. (1996). El análisis de contenido. Madrid: Ediciones Akal.

Barreto, I., \& Borja, H. (2002). Violencia política: algunas consideraciones desde la psicología social. Revista Diversitas- Perspectivas en Psicología, 3(1), 109-119.

Bar-Tal, D., Sharvit, K., Halperin. E., \& Zafran, A. (2012). Ethos of conflict: $f$ e concept and its measurement. Peace and Conflict: Journal of Peace Psychology. 18(1), 40-61.

Bellamy, A. (2009) Guerras justas: de Cicerón a Iraq. Madrid: Fondo de Cultura Económica.

Bizumic, B., Stubager, R., Mellon, M., Van der Linden, N., Iyer, R., \& Jones,. J. (2013). On the (in)compatibility of attitudes toward peace and war. Political Psychology, 34(5) 673-693. doi: 10.1111 pops. 12032

Bobowik, M., Páez, D., Liu, J., Licata, L., Klein, O. \& Basabe, N. (2014). Victorious justifications and criticism of defeated: Involvement of nations in world wars, social development, cultural values, social representations of war, and willingness to fight. International Journal of Intercultural Relations, 43, 60-73.

Bolívar, I. (2006). Discurso emocionales y experiencia de la política: las FARC y las AUC en los procesos de negociación del conflicto (1998-2005). Bogotá, Colombia: Uniandes-Ceso \& cinep.

Borja-Orozco, H., Barreto, I., Sabucedo, J. \& López-López, W. (2008). Construcción del discurso deslegitimador del adversario: gobierno y paramilitarismo en Colombia. Universitas Psychologica, 7(2), 571-583.

Botero, R. (2005). La dimensión retórica y el discurso de Álvaro Uribe Vélez sobre el conflicto armado en Colombia. En Acosta, G. \& Ramírez, L. (2005). Estudios del discurso en Colombia. Medellín: Universidad de Medellín \& Alep. Bothwell, R., \& Kennison, E. (2004). $f$ e authoritarian personality and attitudes toward war. Peace Rewiew, 16(4), 467-470.

Carnagey, N. \& Anderson, C. (2001). Changes in attitudes towards war and violence after September 11, 2001. Aggressive Behavior, 33, 118-129.

Chaux, E., Arboleda , J. \& Rincón, C. (2012). Violencia comunitaria y agresión reactiva y proactiva: el papel mediacional de las variables cognitivas y emocionales. Revista Colombiana de Psicología, 21(2), 233-251.

Cohrs, C., \& Mochner, B. (2002). Antiwar knowledge and generalized political attitudes as determinants of attitude toward the Kosovo War. Peace and Conflict: Journal of Peace Psycho$\log y, 8(2), 139-155$. 
Cohrs, C., Moschner, B., Maes, J., \& Kielman, S. (2005). Personal Values and Attitudes Toward War. Peace and Conflict. Journal of Peace Psychology, 11(3), 293-312.

Cheung-Blunden, B., \& Blunden, B. (2008). The emotional construal of war: Anger, fear, and other negative emotions. Peace and Conflict, 14, 144-149.

Crowson, M. (2009). Right-wing authoritarianism and social dominance orientation as mediators of worldview beliefs on attitudes related to the war on terror. Social Psychology, 40(2), 93-103.

Dunwoody, P., Plane, D., Trescher, S. \& Rice, D. (2014). Authoritarianism, social dominance, and misperceptions of war. Peace and Conflict: Journal of Peace Psychology, 20(3), 256-266.

Echevarría, C., \& Vasco, E. (2006). Justificaciones morales de los bueno y lo malo en un grupo de niñas y nińos provenientes de contextos violentos y no violentos de una ciudad de la zona andina de Colombia. Acta Colombiana de Psicología, 9(1): 51-62.

Friese, M., Fishman, S., Beatson, R., Sauerwein, K., \& Rip,. R. (2009). Whose fault is it anyway? Political orientation, attributions of responsibility, and support for the war in Iraq. Social Justice Research, 22, 280-297.

Gibson, S. (2012). 'I'm not a war monger but...': Discourse analysis and social psychological peace research. Journal of Community and Applied Social Psychology, 22(2), 159-173.

Giroux, S., \& Tremblay, G. (2004). Metodología de las ciencias humanas: la investigación en acción. México, D.F.: Fondo de Cultura Económica.

Halverscheid, S \& Witte, E (2008). Justification of war and terrorism: A comparative case study analyzing ethical positions based on prescriptive attribution theory. Social Psychology, 39(1), 26-36.

Hoffman, M. (2002). Desarrollo moral y empatía. Barcelona: Idea Books Universitaria.

Hogenraad, R. (2005). What the words of war can tell us about the risk of war. Journal of Peace Psychology, 11(2), 137-151.

Jones. G, Restori. A, Howard B. L, Himelfarb. I., \& Boluyt. M. (2007). A psychometric evaluation of existing attitude measures toward capital punishment and war. North American Journal of Psychology, 9(2), 475-484.

De Souza, L., Sperb, T., Mccarthy, S., \& Biaggio,. A. (2006). Children's conceptions of peace, war, and violence. Peace and Conflict: Journal of Peace Psychology, 12(1), 49-63.

Kohlberg, L. (1992). Psicología del desarrollo moral. Bilbao: Editorial Desclée de Brouwer. 
Kuterovac, G. (2000). Is war a good or a bad thing? The attitudes of Croatian, Israeli, and Palestinian children toward war. International Journal of Psychology, 35(6), 241-257.

Li, C., Li, D.-m., Huang, Z., \& Chiu, C. (2015). Peace and war: Rewarding intergroup contacts make past intergroup aggression unforgivable. Peace and Conflict: Journal of Peace Psychology. Advance online publication, 22(2), 166-167.

Lialli , H., \& forsteinsson, E. (2007). Attitudes to the Iraq war and mandatory detention of asylum seekers: Associations with authoritarianism, social dominance, and mortality salience. Australian Journal of Psychology, 59(2), 70-77.

Marulanda, J., \& Yáńez-Canal, J. (2015). Moralidad y justicia en la guerra: reflexiones desde la psicología moral. En J. Yáñez-Canal, J. Chaparro, \& L. Segovia (eds.) Justicia guerra y mundo social. Bogotá: Uniminuto.

Mayton. D. (1988). Measurement of nuclear war attitudes: methods and concerns. Basic and Applied Social Psychology, 9(4), 241-263.

Moerk, E. (2002). Scripting war-entry to make it appear unavoidable. Peace and Conflict: Journal of Peace Psychology, 8(3), 229-248.

Musgrove. L., \& McGarty. C. (2008). Opinion-based group membership as a predictor of collective emotional responses and support for pro- and anti-war action. Social Psychology, 39(1), 37-47.

Myers-Bowman, K., Walker, K., \& Myers-Walls, J. (2005). "Differences between war and peace are big": Children from Yugoslavia and the United States describe peace and war. Peace and Conflict: Journal of Peace Psychology, 11(2), 177-198.

Nasie, M. \& Bar-Tal, D. (2012). Sociopsychological infrastructure of an intractable conflict through the eyes of Palestinian children and adolescents. Peace and Conflict: Journal of Peace Psychology, 18(1), 3-20.

Orend, B. (2006). The morality of war. Toronto: Broadview Press.

Papacchini, A. (2002). La Ética ante el Desafio de La Guerra. Cali: Universidad del Valle. Piaget, J. (1999). The moral judgment of the child. Nueva York: Routledge \& Kegan Paul Ltd.

Pilecki, A., Muro, J., Hammack, P., \& Clemons, C. (2014). Moral exclusion and the justification of U.S. Counterterrorism Strategy: Bush, Obama, and the terrorist enemy figure. Peace and Conflict: Journal of Peace Psychology, 20(3), 285-299.

Posada, R. (2012). Experiencias de violencia y razonamiento moral en un contexto de venganza. Revista Colombiana de Psicología, 21(2), 197-212. 
Posada. A, \& Wainryb, C. (2007). Moral development in a violent society: Colombian children's judgments in the context of survival and revenge. Child Development, 79, 882-898.

Riaño, E. (2002). Socialización y carreras morales en jóvenes desvinculados del conflicto armado. Tesis Psicológica, 1, 41-51.

Sarrica, M., \& Wachelke, J. (2009). Peace and war as social representations: A structural exploration with Italian adolescents. Universitas Psychologica, 9(2), 315-330.

Schroeder. D., Gaier, E., \& Holdnack. J. (1993). Middle adolescents' views of war and American military involvement in the Persian Gulf. Adolescense, 28(11), 950.

Shaw, M., Quezada, E., \& Zárate, M. (2011). Violence with a conscience: Religiosity and moral certainty as predictors of support for violent warfare. Psychology of Violence, 11(4), 275-286.

Sundberg, R. (2014). Violent values: Exploring the relationship between human values and violent attitudes. Peace and Conflict: Journal of Peace Psychology, 20(1), 68-83.

Van der Linden, N., Bizumic, B., Stubager, R. \& Mellon, S. (2011). Social representational correlates of attitudes toward peace and war: A cross-cultural analysis in the United States and Denmark. Peace and Conflict: Journal of Peace Psychology, 17(3), 217-242.

Walzer, M. (2001a). Guerras justas e injustas. Barcelona: Paidós. Walzer, M. (2001b). Guerra, política y moral. Barcelona: Paidós.

Wainryb, C. \& Pasupathi, M. (2008). Developing Moral Agency in the Midst of Violence: Children, Political Conflict, and Values. En B. Karawan, W. McCormack, \& S. Reynolds (eds.) Values and violence: untangible aspect of terrorism (pp. 169-187). New York: Springer.

Wille, W. (2007). Ambivalence in the Christian attitude to war and peace. International Review of Psychiatry, 19(3), 235-242.

Yáñez-Canal , J. (2000). Debates en la psicología del desarrollo moral. En E. Aguirre, \& J. Yáñez- Canal (eds.) Diálogos: Discusiones en la psicología contemporánea, Número 1. Bogotá: Universidad Nacional de Colombia.

Yáñez-Canal. J., Perdomo. A., \& Mojica. A. (2010). La moral, la psicología del desarrollo y la teoría de los dominios. En J.M. Jaramillo \& T. Bernal (Comp.), La Infancia En La Sociedad Actual (pp. 109-138). Bogotá, Colombia: Universidad Santo Tomas.

Yáñez-Canal. J. \& Mojica. A. (2012). Kohlberg y su teoría cognitiva de lo moral: algunas inconsistencias y limitaciones de su modelo formal. En J. Yáñez-Canal, J. Chaparro, \& L. Segovia (eds.) Experticia, sabiduría y desarrollo moral (pp. 67-112). Bogotá: Uniminuto. 